\title{
Mapping the Characteristics of Critical Care Facilities: Assessment, Distribution, and Level of Critical Care Facilities from Central India
}

\begin{abstract}
Saurabh Saigal, Jai Prakash Sharma', Abhijit Pakhare ${ }^{2}$, Santosh Bhaskar ${ }^{3}$, Sanjay Dhanuka ${ }^{4}$, Sanjay Kumar ${ }^{5}$, Yogesh Sabde ${ }^{6}$, Pradip Bhattacharya ${ }^{7}$, Rajnish Joshi ${ }^{8}$ Department of Trauma and Emergency Medicine, AllMS, Departments of ${ }^{1}$ Anaesthesia and ${ }^{2} \mathrm{CFM}$, AllMS, ${ }^{3}$ Department of Anaesthesia, CMCH, Bhopal, ${ }^{4}$ Department of CCM, Greater Kailash Hospital, Indore, Madhya Pradesh, ${ }^{5}$ Department of Anaesthesia, ANNIMS, Port Blair, Andaman and Nicobar Islands, ${ }^{6}$ Scientist E, NIREH, ${ }^{7}$ Department of Emergency Medicine and CCM, CMCH, ${ }^{8}$ Deparment of Medicine, AlIMS, Bhopal, Madhya Pradesh, India
\end{abstract}

\section{Abstract}

Background: In low- and middle-income countries such as India, where health systems are weak, the number of available Critical Care Unit (Intensive Care Unit [ICU]) beds is expected to be low. There is no study from the Indian subcontinent that has reported the characteristics and distribution of existing ICUs. We performed this study to understand the characteristics and distribution of ICUs in Madhya Pradesh (MP) state of Central India. We also aimed to develop a consensus scoring system and internally validate it to define levels of care and to improve health system planning and to strengthen referral networks in the state. Methods: We obtained a list of potential ICU facilities from various sources and then performed a cross-sectional survey by visiting each facility and determining characteristics for each facility. We collected variables with respect to infrastructure, human resources, equipment, support services, procedures performed, training courses conducted, and in-place policies or standard operating procedure documents. Results: We identified a total of 123 ICUs in MP. Of 123 ICUs, 35 were level 1 facilities, 74 were level 2 facilities, and only 14 were level 3 facilities. Overall, there were 0.17 facilities per 100,000 population (95\% confidence interval [CI] $0.14-0.20$ per 100,000 populations). There were a total of 1816 ICU beds in the state, with an average of 2.5 beds per 100,000 population (95\% CI 2.4-2.6 per 100,000 population). Of the total number of ICU beds, 250 are in level 1, 1141 are in level 2 , and 425 are in level 3 facilities. This amounts to $0.34,1.57$, and 0.59 ICU beds per 100,000 population for levels 1,2 , and 3 , respectively. Conclusion: This study could just be an eye opener for our healthcare authorities at both state and national levels to estimate the proportion of ICU beds per lac population. Similar mapping of intensive care services from other States will generate national data that is hitherto unknown.

Keywords: Critical care units, India, Intensive Care Unit scoring, levels of Intensive Care Unit, Madhya Pradesh

\section{INTRODUCTION}

In developed countries, caring for critically ill patients involves a coordinated system of triage, emergency management, and critical care. This is complicated and unaffordable for many low-income countries. In contrast, critical care remains at a very nascent stage in many low-income countries. ${ }^{[1]}$ The burden of critical illness is especially high in low-income countries, and the spectrum of illnesses is wide-ranging from tropical infections, sepsis, airway diseases, vascular diseases, and road traffic accidents to obstetrical and surgical emergencies. ${ }^{[2]}$ Intensive care units (ICUs) are specialized facilities that provide care for patients with severe life-threatening illnesses. ${ }^{[3]}$ Further, critically ill patients have extremes of human physiology and a higher risk of mortality;

\begin{tabular}{|l|l|}
\hline \multicolumn{3}{|c|}{ Access this article online } \\
\hline Quick Response Code: & Website: \\
& www.ijccm.org \\
& \\
&
\end{tabular}

hence, critical care physicians and nursing staff need more skills and training. Given high demands of this essential component of health system, nations need to devote more resources for creation of ICUs. ${ }^{[4]}$

There is a paucity of information on organization of critical care facilities in low-income countries. ${ }^{[5-7]}$ In a low- and middle-income country (LMIC) such as India, where health

\section{Address for correspondence: Dr. Saurabh Saigal Department of Trauma and Emergency Medicine, AllMS, Bhopal, Madhya Pradesh, India. E-mail: saurabh.criticalcare@aiimsbhopal.edu.in}

This is an open access article distributed under the terms of the Creative Commons Attribution-NonCommercial-ShareAlike 3.0 License, which allows others to remix, tweak, and build upon the work non-commercially, as long as the author is credited and the new creations are licensed under the identical terms.

For reprints contact: reprints@medknow.com

How to cite this article: Saigal S, Sharma JP, Pakhare A, Bhaskar S, Dhanuka S, Kumar S, et al. Mapping the characteristics of critical care facilities: Assessment, distribution, and level of critical care facilities from central India. Indian J Crit Care Med 2017;21:625-33. 
systems are weak, the number of available ICU beds is expected to be low. There is no study from the Indian subcontinent that has reported the characteristics and distribution of existing ICUs. We performed this study to understand the characteristics and distribution of ICUs in Madhya Pradesh (MP) state of Central India. We also aimed to describe the heterogeneity in different types of ICUs and to classify ICUs according to the level of care in the state of MP. Various critical care professional bodies have defined levels of ICU care based on set of services and functionalities available within a facility. During our pilot study, we found that many facilities had functionalities corresponding to a higher level, yet lacking in functionality that was expected at a lower level of ICU care. Hence, we also aimed to develop a consensus scoring system and internally validate it to define levels of care.

\section{Methods}

\section{Setting}

MP is the second largest $\left(308,000 \mathrm{~km}^{2}\right)$ and fifth most populous (72 million) state of India. ${ }^{[8]}$ It has 51 districts as its administrative units. The Chief Medical Health Officer (CMHO) in the district supervises public health delivery system. In addition, there are a number of private hospitals and nursing homes in the state. There are two professional bodies that maintain a list of private care facilities (MP Nursing Home Association) and critical care practitioners (Indian Society of Critical Care Medicine [ISCCM]). However, none of these lists is comprehensive.

\section{Study design and permissions}

We performed a cross-sectional survey by visiting each facility and determining characteristics for each facility. The Ethics Committee at AIIMS Bhopal approved the study protocol. We obtained permission for the survey from the Department of Health and Family Welfare, Government of MP. A verbal consent was obtained from $\mathrm{CMHO}$ or in-charge of each facility before facility assessment.

\section{Sources of information}

We obtained a list of potential ICU facilities from various sources. First, a list of all teaching medical institutes was obtained from the Medical Council of India's online database, all of which have an ICU. ${ }^{[9]}$ Second, we contacted CMHO of each district to identify any additional ICU facilities in the district. Third, we obtained a list of private nursing homes and hospitals from MP Private Hospital and Nursing Home Association and contacted each one of them to identify if these had an ICU. Fourth, we contacted members of ISCCM in the state to enlist ICU facilities they were aware about. Fifth, we asked doctors in government as well as private sector if they were aware of any ICU in their districts. Finally, we obtained a list of potential facilities from representatives of manufacturers of high-end equipment such as ventilators, monitors, and hemodialysis machine. District-level indicators were extracted from www.data.gov.in, a Government of India's open data platform portal and Census 2011. ${ }^{[10]}$

\section{Selection of intensive care units}

Trained field investigators visited each district of the state enlisted all potential ICU facilities in the district from the multiple information sources available. Thereafter, field investigators visited each of the identified facilities to identify hospitals that had a functional ICU. We defined hospital as a physical facility consisting of one or more buildings in a contiguous area, where professionally trained medical professionals perform patient-care activities (including a facility to admit patients for $24 \mathrm{~h}$ or longer). We defined ICU as a physical area inside a hospital where it is possible to provide advanced organ support to patients. Measures that can provide advanced organ support include mechanical ventilator (lung support), hemodialysis (renal support), and intra-aortic balloon pump (cardiac support). Availability of a functional invasive mechanical ventilator was essential for a facility to be designated as an ICU. Each identified ICU was approached for participation in the study and consent was sought from the facility in-charge for facility assessment. We collected geographic coordinates for each eligible and consenting ICU as a part of mapping activity. We have not enrolled pediatric ICUs and neonatal ICUs in our study. We have included postoperative ICU, postcardiac surgery ICU, and postneurosurgery ICUs in our study. All of the level 3 ICUs had separate postoperative cardiac surgery ICU while postoperative patients, neurosurgery patients, and poisoned patients were kept in general medical ICU.

\section{Facility assessment and scoring}

Facility assessment had two components, first was a self-reported information about facilities by the facility in-charge. Thereafter, key variables were observed and verified by the field investigators. We collected variables with respect to infrastructure, human resource, equipment, academic activity, and ancillary services such as radiology, laboratory services, blood bank services, ambulance services, and other services [Table 1].

\section{Infrastructure}

Infrastructure of facilities were assessed in terms of type of ICUs open versus closed, number of beds, bed space, pre-ICU triage, post-ICU stepdown, isolation facility, and availability of 24-h ambulance services.

\section{Human resources}

Human resources were assessed in different cadre required to run ICU as availability of qualified intensivist or doctors other than qualified intensivist, availability of nurses and support staff as per bed, ICU technician, and full-time physiotherapist.

\section{Equipment}

Equipment was assessed in two categories basic equipment and high-end equipment required in relation to level of care catered in ICU. Basic equipment includes ratio of oxygen-suction air ports on bed-head panel of medical care gas pipeline, arterial blood gas (ABG) analyzer, critical care monitors, per ICU bed ratio of invasive or noninvasive ventilator, and defibrillator. 


\section{Table 1: Facility assessment checklist: Critical care facility}

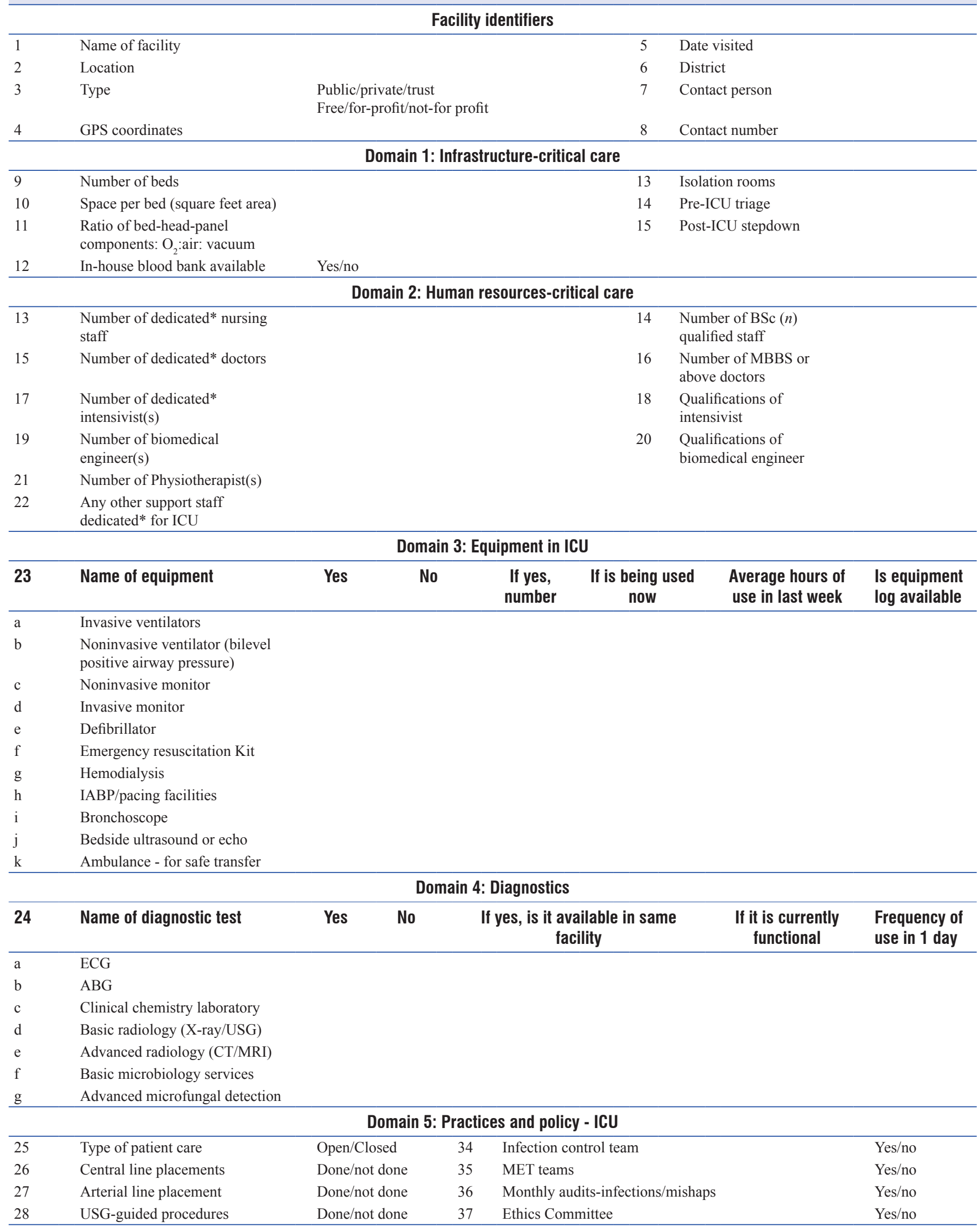




\begin{tabular}{|c|c|c|c|c|c|}
\hline \multicolumn{6}{|c|}{ Domain 5: Practices and policy - ICU } \\
\hline 29 & $\begin{array}{l}\text { Percutaneous tracheostomy } \\
\text { placement }\end{array}$ & Done/not done & 38 & $\begin{array}{l}\text { Any certified training courses being run for nurses/ } \\
\text { technicians }\end{array}$ & Yes/no \\
\hline 30 & PA catheters placement & Done/not done & 39 & Any certified training courses being run for doctors & Yes/no \\
\hline 31 & Transcutaneous pacing & Done/not done & 40 & Is there a written ICU protocol & Yes/no \\
\hline 32 & Hemodialysis catheters placement & Done/not done & 41 & Library & Yes/no \\
\hline 33 & $\begin{array}{l}\text { Using advanced hemodialysis } \\
\text { gadgets-PiCCO, Flotrac }\end{array}$ & Done/not done & 42 & Organ donation facility/protocol & Yes/no \\
\hline
\end{tabular}

\begin{abstract}
*Human resource that is responsible only for the ICU facility being assessed and no other is defined as a dedicated human resource. ICU: Intensive Care Unit; MRI: Magnetic resonance imaging; USG: Ultrasonography; CT: Computed tomography; IABP: Intra-aortic balloon pump; MET: Medical Emergency Team; PiCCO: Pulse Contour Cardiac Output; ABG: Arterial blood gas; ECG: Electrocardiogram; PA: Pulmonary artery
\end{abstract}

Specialized equipment includes dedicated Echocardiography, fiberoptic bronchoscope, hemodialysis or continuous renal replacement therapy (CRRT), and cardiac output monitor.

\section{Ancillary services}

They include pathology, biochemistry, imaging (ultrasonography [USG], computed tomography [CT], magnetic resonance imaging [MRI]), echocardiography, basic and advanced microbiology, and blood bank.

\section{Academic activity}

a. Whether assessed ICUs are running regular teaching, training program, short duration courses of 3 months to 1 year (such as fellowships [by ISCCM] or Post-Doctoral Certificate Course), super-specialization in critical care, 3-year or 2-year course DM/FNB

b. ICU procedures: Whether routine ICU procedures such as arterial cannulation, invasive pressure monitoring, central venous cannulation, central venous pressure monitoring, and pleural tapping and high-end ICU procedures such as echocardiography examination, percutaneous tracheostomy, hemodialysis catheter insertions, and chest tube insertion are performed

c. Research work and written operation procedures: These are mandatory for quality care and upgradation of care such as standing operating procedures of routine ICU activity or common diseases, written disinfection policies, seminar or journal club schedule, and ethics committee.

A definition of some key indicators is provided in Table 2. We identified 15 key indicators that could discriminate between levels of ICU. Item content-validity index was calculated for each item. For this, we contacted 10 critical-care providers across India and asked them to rate each indicator as "very relevant," "relevant," "somewhat relevant," and "not relevant." Then pooling "very relevant" and "relevant" in one category i.e. "Relevant". "Somewhat relevant" and "Not Relevant" in another category i.e. "Not Relevant" which generated dichotomous response. Items with $>80 \%$ of "relevant" responses were shortlisted for final scoring scale. Ten such items were identified. Each of these indicators was equally weighted and scored from 1 to 3 . The facilities that had a score from 5 to 10 were designated as level 1,11 to 20 as level 2, and 21 or more as level 3 [Table 3].

\section{Statistical analysis}

We analyzed distribution of facilities by district. Facility density per 100,000 population with its $95 \%$ CI was calculated and map was generated using Q-GIS software. We estimated the total number of ICU beds available in every district. To identify which district-level indicators were associated with higher number of ICU beds, we performed linear regression with number of ICU beds as an outcome variable and macroindicators (population, area, per-capita income, literacy, population of individuals belonging to scheduled tribes and scheduled castes) as explanatory variables. Variables whose 95\% confidence interval (CI) of beta-coefficient did not include zero (corresponding to significance level of $<0.05$ ) were considered as significant predictors of more ICU beds in the district.

We computed Cronbach's alpha for testing internal consistency of ten-item facility scoring system. We described distribution of facility-level variables by level of facility. Some of these variables that were not included in the scoring system (median bed space in square feet [range], presence of isolation room, pre-ICU triage, post-ICU stepdown, ambulance facility, median number of doctors, availability of an intensivist, median number of nursing staff, presence of medical emergency team, and hand rub availability at each bed) were evaluated to determine internal validity of the scoring system. We evaluated if there was a significant trend for these variables across facility levels from level 1 (lowest facility level) to level 3 (highest facility level) using Chi-square test for categorical variables and Kruskal Wallis test for numeric variables (significance level $P<0.05$ ) for categorical variables and Kruskal-Wallis test for numerical variables.

\section{RESULTS}

We conducted facility assessment survey between March and December 2015 in 49 of the 50 districts in the state and identified a total of 123 ICUs - of those 98 were open ICU and 25 were closed ICU. Ten-item facility scoring had high internal consistency with Cronbach's alpha of 0.833 . Of 123 ICUs, 35 were level 1, 74 were level 2, and 14 were level 3 facilities [Table 4]. Overall, $85(75 \%)$ facilities were private for-profit, $9(8 \%)$ were private not-for-profit, and 19 (17\%) were public sector facilities. Overall, there were 0.17 facilities 
Table 2: Facility indicators and definitions

Facility indicator

Number of critical care beds

Nurse-to-bed ratio

Invasive ventilator-to-bed ratio

Doctor in-charge of critical care facility

Monitoring devices

Imaging facilities

Laboratory facilities

Level of procedures conducted

Policies and protocols

Teaching and training facilities

\section{Definition}

A critical care bed was defined as one located inside a critical care facility that had a head-end panel with at least one port for oxygen, suction, or medical air and a bedside monitoring device Nurse-to-bed ratio was calculated based on average number of staff nurses in each shift, per critical care bed in the facility

Invasive ventilator-to-bed ratio was calculated based on total number of functional invasive ventilators available in the facility per critical care bed

A medically qualified person who has overall responsibility for individual treatment decisions and coordination across other specialties

Devices inside a critical care facility that can measure, record and display biological parameters on a continual basis are defined as monitoring devices. These largely include bed-side monitors for heart rate, respiratory rate, pulse oximetry, noninvasive blood pressure, and invasive pressure monitoring. Imaging devices inside the hospital premises that help visualize organ systems that include radiography, USG, CT, and MRI scans

Laboratory facilities inside the hospital premises that can test biological fluids (such as blood, plasma, serum, CSF, and secretions) These include pathology, biochemistry and microbiology testing facilities. Microbiology facility where fungal cultures are performed is defined as an advanced microbiology facility

Procedures that are performed inside the critical care facility, either to provide a greater access to blood vessels, airways, stomas, or body cavities

Policies and protocols include written and printed statements customized for the critical care facility that provide instructions and guidelines for medical staff for facility and patient management. These include standard operating protocols, ethics statements, and infection control guidelines

Teaching and training facilities include formal recognized training programs of 6 months or longer in duration that have an evaluation mechanism and result in awarding of a certificate or a diploma

Open ICU: If final orders are written by some other specialist then it is called open ICU; Closed ICU: An ICU where the final orders were written by the intensive care team. ICU: Intensive Care Unit; MRI: Magnetic resonance imaging; CSF: Cerebrospinal fluid; USG: Ultrasonography; CT: Computed tomography

\section{Table 3: Scoring grid for level of care of Intensive Care Unit's}

\begin{tabular}{|c|c|c|c|}
\hline \multirow[t]{2}{*}{ Parameters } & \multicolumn{3}{|c|}{ Score } \\
\hline & 1 & 2 & 3 \\
\hline Number of ICU beds & 6 or less & $7-12$ & 12 or more \\
\hline Nurse to patient ratio & $1: 4$ or less & $1: 3$ & $1: 2$ or more \\
\hline Invasive ventilator to bed ratio & $1: 4$ or less & $1: 3-1: 2$ & $1: 1$ or more \\
\hline $\begin{array}{l}\text { Qualification of doctor in } \\
\text { charge of critical care facility }\end{array}$ & $\begin{array}{l}\text { MBBS or } \\
\text { post-MBBS diploma }\end{array}$ & MD or MS & Post-MD/MS critical care qualification \\
\hline $\begin{array}{l}\text { Monitoring devices and } \\
\text { advanced gadgets }\end{array}$ & $\begin{array}{l}\text { Noninvasive } \\
\text { monitors }\end{array}$ & $\begin{array}{l}\text { Noninvasive and invasive } \\
\text { monitors }\end{array}$ & $\begin{array}{l}\text { Noninvasive and invasive monitors and advanced } \\
\text { gadgets (hemodialysis, IABP, advanced ventilators etc.) }\end{array}$ \\
\hline Imaging facilities & Radiography/USG & $\begin{array}{l}\text { Radiography/USG CT and } \\
\text { echocardiography }\end{array}$ & Radiography/USG CT and echocardiography \\
\hline Laboratory facilities & $\begin{array}{l}\text { Pathology and } \\
\text { clinical chemistry }\end{array}$ & $\begin{array}{l}\text { Pathology and clinical chemistry } \\
\text { and basic microbiology }\end{array}$ & $\begin{array}{l}\text { Pathology and clinical chemistry and basic and } \\
\text { advanced microbiology }\end{array}$ \\
\hline $\begin{array}{l}\text { Level of procedures } \\
\text { conducted }\end{array}$ & $\begin{array}{l}\text { Peripheral and central } \\
\text { venous cannulation }\end{array}$ & Venous and arterial cannulation & $\begin{array}{l}\text { Venous, arterial, and cannulation and percutaneous } \\
\text { tracheostomy }\end{array}$ \\
\hline Policies and protocols & $\begin{array}{l}\text { Standard operating } \\
\text { protocols }\end{array}$ & $\begin{array}{l}\text { Standard operating protocol and } \\
\text { infection control policy }\end{array}$ & $\begin{array}{l}\text { Standard operating protocol, infection control policy, } \\
\text { and ethics committee }\end{array}$ \\
\hline $\begin{array}{l}\text { Teaching and training } \\
\text { facilities }\end{array}$ & $\begin{array}{l}\text { Short-term courses } \\
\text { (up to } 6 \text { months) }\end{array}$ & $\begin{array}{l}\text { Indian diploma in critical care } \\
\text { (up to } 1 \text { year) }\end{array}$ & DM in critical care (3-year course) \\
\hline
\end{tabular}

Score: 5-10 (Level I); 11-20 (Level II); 21-30 (Level III). IABP: Intra-aortic balloon pump; USG: Ultrasonography; CT: Computed tomography; DM: Doctorate of Medicine

per 100,000 population (95\% CI $0.14-0.20$ per 100,000 population). There were a total of 1816 ICUs beds in the state, with an average of 2.5 beds per 100,000 population (95\% CI 2.4-2.6 per 100,000 population). Of the total number of ICU beds, 250 are in level 1, 1141 are in level 2, and 425 are in level 3 facilities. This amounts to $0.34,1.57$, and 0.59 ICU beds per 100,000 population for levels 1,2 , and 3 , respectively [Table 4].
Availability of human resources in different cadres like the qualified intensivist as defined in Table 1 were present round the clock in 17/123 (13.8\%) facilities, 92/123 facilities they were present on call and in 14/123 facilities no intensivist was available. The nursing staff was available in ICU round the clock, but nurse-to-patient ratio varied in different levels of ICU care - median number of nurses was 5, 14.5, and 43 


\begin{tabular}{|c|c|c|c|c|}
\hline Variable & Level 1 & Level 2 & Level 3 & $P$ value for trend \\
\hline$n$ & 35 & 74 & 14 & \\
\hline \multicolumn{5}{|l|}{ Infrastructure } \\
\hline Total ICU beds & 250 & 1141 & 425 & \\
\hline Median number of ICU beds & 6 & 12 & 24 & NA \\
\hline Median bed space in square feet (range) & 89.14 & 102.6 & 126.4 & 0.000003 \\
\hline Isolation room & $42.9 \%$ & $78.4 \%$ & $92.9 \%$ & 0.000086 \\
\hline Pre-ICU triage & $22.9 \%$ & $67.6 \%$ & $100.0 \%$ & 0.0000006 \\
\hline Post-ICU step down & $37.1 \%$ & $74.3 \%$ & $100.0 \%$ & 0.0000054 \\
\hline Ambulance & $74.3 \%$ & $97.3 \%$ & $100.0 \%$ & 0.000605 \\
\hline \multicolumn{5}{|l|}{ Human resources } \\
\hline Median number of doctors & 3 & 5 & 13.5 & $<0.0000001$ \\
\hline Intensivist availability & $68.6 \%$ & $97.3 \%$ & $100.0 \%$ & 0.00006881 \\
\hline Median number of nursing staff & 5 & 14.5 & 43.5 & $<0.0000001$ \\
\hline Medical emergency team & $0.0 \%$ & $9.5 \%$ & $92.9 \%$ & $<0.0000001$ \\
\hline Hand rub availability at each bed & $5.7 \%$ & $64.9 \%$ & $100.0 \%$ & $<0.0000001$ \\
\hline Nurse:bed ratio & $1: 3$ & $1: 2.7$ & $1: 1.7$ & NA \\
\hline \multicolumn{5}{|l|}{ Equipment availability } \\
\hline Median number of ventilators & 2 & 4 & 14 & \\
\hline Ventilator:bed ratio & $1: 4$ & $1: 3$ & $1: 2$ & NA \\
\hline BiPAP & $45.7 \%$ & $89.2 \%$ & $100.0 \%$ & NA \\
\hline Noninvasive monitors & $97.1 \%$ & $94.6 \%$ & $100.0 \%$ & NA \\
\hline Invasive monitors & $5.7 \%$ & $62.2 \%$ & $100.0 \%$ & NA \\
\hline Hemodialysis & $22.9 \%$ & $70.3 \%$ & $100.0 \%$ & NA \\
\hline Bronchoscope & $2.9 \%$ & $47.3 \%$ & $92.9 \%$ & NA \\
\hline Echocardiography & $22.9 \%$ & $77.0 \%$ & $100.0 \%$ & NA \\
\hline \multicolumn{5}{|l|}{ Laboratory/investigations } \\
\hline $\mathrm{ABG}$ & $22.9 \%$ & $90.5 \%$ & $100.0 \%$ & NA \\
\hline CT scan & $0.0 \%$ & $40.5 \%$ & $100.0 \%$ & NA \\
\hline Blood bank & $28.6 \%$ & $35.1 \%$ & $71.4 \%$ & NA \\
\hline Basic microbiology laboratory & $2.9 \%$ & $64.9 \%$ & $100.0 \%$ & NA \\
\hline Advanced microbiology laboratory & $0.0 \%$ & $8.1 \%$ & $78.6 \%$ & NA \\
\hline \multicolumn{5}{|l|}{ Procedures } \\
\hline Central venous catheter placement & $34.3 \%$ & $98.6 \%$ & $100.0 \%$ & NA \\
\hline Arterial line placement & $0.0 \%$ & $48.6 \%$ & $100.0 \%$ & NA \\
\hline USG-guided procedures & $0.0 \%$ & $37.8 \%$ & $100.0 \%$ & NA \\
\hline \multicolumn{5}{|l|}{ Training courses } \\
\hline Training courses for nurses & $0.0 \%$ & $2.7 \%$ & $28.6 \%$ & NA \\
\hline Training course for doctors & $0.0 \%$ & $0.0 \%$ & $7.1 \%$ & NA \\
\hline \multicolumn{5}{|l|}{ Policies } \\
\hline ICU protocol & $0.0 \%$ & $18.9 \%$ & $100.0 \%$ & NA \\
\hline Infection control & $0.0 \%$ & $37.8 \%$ & $100.0 \%$ & NA \\
\hline Ethics committee & $0.0 \%$ & $9.5 \%$ & $50.0 \%$ & NA \\
\hline
\end{tabular}

ABG: Arterial blood gas; ICU: Intensive Care Unit; NA: Not available; USG: Ultrasonography; CT: Computed tomography; BiPAP: Bilevel positive airway pressure

in level 1, 2, 3 ICUs. A dedicated infection control nurse was present in $1 / 3^{\text {rd }}$ of ICU setups. ICU technicians were present in $24 \%$ of the ICUs while full-time physiotherapists were present in $19 \%$ of the ICUs [Table 5].

Specialized equipment such as ABG machine was present in $88 / 123$ of ICU setups, hemodialysis in $73 / 123$ of setups, whereas CRRT was present in $8 / 123$ of setups. The portable USG machine with echocardiography was present in 43/123 of ICU setups, i.e., mostly in level 2 and level 3 facilities.
The CT scan was present in 44/123 of ICU setups whereas MRI was present in 17/123 of ICUs. The clinical chemistry and hematology services were present in almost all setups, but microbiology services were present in $64 / 123$ of ICU setups [Table 5].

Of the ten variables used to test the internal validity of the facility scoring system, all demonstrated significant differences in distribution and increasing trend with respect to facility level. Services those were not present in all the level 3 ICUs 


\begin{tabular}{|c|c|c|c|}
\hline & $\begin{array}{l}\text { Available in } \\
\text { ICU (round } \\
\text { the clock) }\end{array}$ & $\begin{array}{l}\text { Available } \\
\text { in hospital }\end{array}$ & NA \\
\hline Qualified/experience physician & $17 / 123$ & $92 / 123$ & $14 / 123$ \\
\hline Nursing: bed ratio (\%) & 100 & NA & NA \\
\hline $\begin{array}{l}\text { Dedicated infection control } \\
\text { nurse }\end{array}$ & $41 / 123$ & NA & NA \\
\hline ICU technician & $30 / 123$ & NA & NA \\
\hline Physiotherapist & $23 / 123$ & $49 / 123$ & \\
\hline Chest X-ray & $5 / 123$ & $114 / 123$ & $4 / 123$ \\
\hline Blood gas analyzer & $88 / 123$ & & $35 / 123$ \\
\hline $\begin{array}{l}\text { Ultrasound (excluding } \\
\text { echocardiography) }\end{array}$ & $43 / 123$ & $76 / 123$ & $4 / 123$ \\
\hline Echocardiography & $43 / 123$ & $37 / 123$ & $43 / 123$ \\
\hline Hemodialysis & $14 / 123$ & $59 / 123$ & $50 / 123$ \\
\hline $\begin{array}{l}\text { Continuous renal replacement } \\
\text { therapy }\end{array}$ & - & $8 / 123$ & $115 / 123$ \\
\hline Fiberoptic bronchoscope & $5 / 123$ & $45 / 123$ & $73 / 123$ \\
\hline Defibrillator/pacer & $94 / 123$ & $29 / 123$ & \\
\hline \multicolumn{4}{|l|}{ Lab services } \\
\hline $\begin{array}{l}\text { Biochemistry/hematology } \\
\text { laboratory }\end{array}$ & & $123 / 123$ & \\
\hline Microbiology services & & $64 / 123$ & $59 / 123$ \\
\hline Computed tomography & & $44 / 123$ & \\
\hline Magnetic resonance imaging & & $17 / 123$ & \\
\hline $\begin{array}{l}\text { Cardiac catheterization } \\
\text { laboratory }\end{array}$ & & $14 / 123$ & \\
\hline Blood bank services & & $47 / 123$ & $76 / 123$ \\
\hline
\end{tabular}

ICU: Intensive Care Unit; NA: Not available

included isolation room, in-house blood bank, advanced microbiology laboratory, bronchoscopy, ethics committee, and training courses (diploma or degree) for doctors or nurses. There was lack of training courses, written operation procedures, policy documents, and medical emergency team from most level 2 facilities as well. While all facilities were equipped for mechanical ventilation and were performing invasive procedures, less than two-thirds of all level 2 facilities and $<10 \%$ of level 1 facilities had hand-rubs available at the bedside [Table 4].

Of the 49 districts in the state, 30 districts had no ICU facility. Of the remaining 19 districts with one or more facility, more than two-thirds of all facilities (86/123 [69\%]) were in four districts (Indore, Bhopal, Jabalpur, and Gwalior). The heterogeneity in distribution of ICU facilities across the state is shown in Figure 1.

\section{Discussion}

There is no singular list of ICUs in India. Further, the definition of an ICU varies from country to country. ${ }^{[1]}$ What may constitute a critical care bed in one country may be considered a stepdown or high-dependency bed in another. This is so as some countries seem to focus on the nurse-to-patient ratio, or need for close monitoring, whereas others focus on the ability to support organ systems. ${ }^{[1]}$ We based our definition

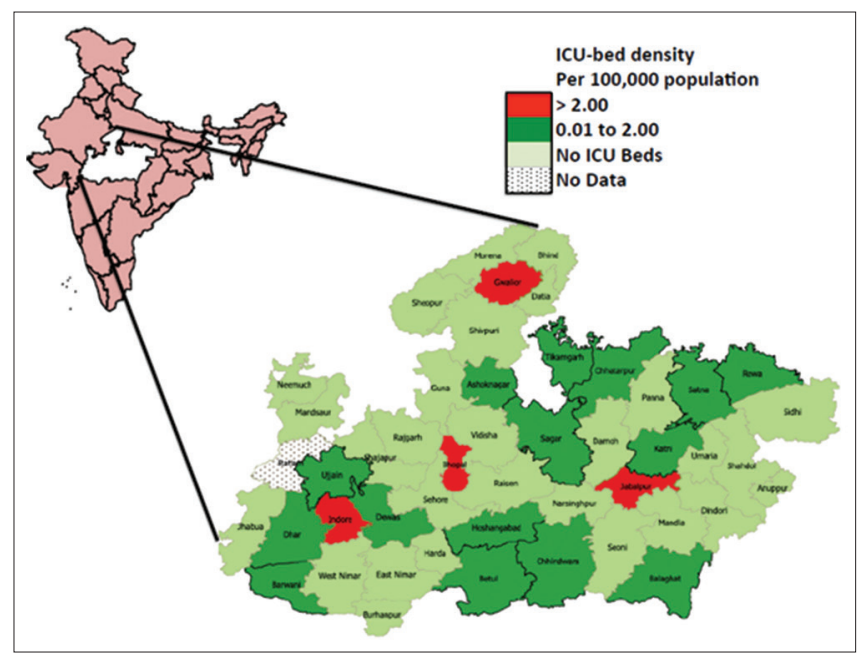

Figure 1: Location of facilities by GPS coordinates

of ICU on ISCCM document that considers a level 1 ICU as a facility where patients can be ventilated for 24-48 h. ${ }^{[12]}$ Hence, we defined ICU as a facility that is able to at least provide mechanical ventilation. We used this minimalistic definition of ICU that leaned toward over-inclusion of eligible facilities. Hence, some level 1 facilities in this study may have lower standards as compared to what may be acceptable in other countries. Thus, our ICU density is more likely to be an overestimate.

While ISCCM has provided a guideline to stratify ICUs as level 1,2 , and 3, we in pilot phase of this study found it difficult to implement. ${ }^{[12]}$ There was number of ICUs that did not have facilities such as in-house blood banks, advanced microbiology, and advanced imaging yet had services such as invasive monitors, hemodialysis, and better nurse-bed ratio. Hence, we developed a consensus scoring of facilities to stratify these into levels of care [Table 2]. We believe this to be a more objective method, which can be applied widely. This scoring was internally valid as criteria not in the score also had a significant trend across levels of care. We need to externally validate this score for better applicability. This facility scoring gives an equal weightage to human resources, equipment, and ancillary services, which may be debatable. We do not know if one of these components should have a higher weightage to define a better ICU.

In the current study, we found that penetration of ICUs and availability of ICU beds is low in the state. There are about 2.5 ICU beds for every 100,000 population, three-fourths of these are in for-profit private sector (hence, reduced accessibility for the poor), and almost nine out of every ten facilities is either level 1 or 2 (hence, lower quality). About $70 \%$ of all ICUs in the state are located in four districts that constitute only $14 \%$ of the total population. For comparison, state of MP is similar in size and population density to Italy, a country that has four times the ICU beds and yet ranks tenth in per-capita ICU beds among all European countries. ${ }^{[4]}$ The results of this study are important not only for future planning 
of ICU services but also because this is first such description of ICUs in an LMIC setting.

Germany has about 24.5 ICU beds per 100,000 population, the United States has 20, France has 8, and the United Kingdom has 3.5. ${ }^{[1]}$ These estimates are from publically funded facilities in these countries and do not include private care. In the current study, state of MP has 2.5 ICU beds for 100,000 (only $25 \%$ of these are in public sector). Factors responsible for this variation are different definitions, variable population size, per-capita income; proportion of GDP spent on health; and ratio of ICU beds to acute care beds in a facility. ${ }^{[4,11]}$ Countries in Europe that spend about 5\% of GDP on health have about 10 ICU beds per 100,000 populations. Critical care experts have recommended ICU beds to be at least $5 \%$ of hospital beds (about 5-6 beds in a 100-beded hospital). India spends $4 \%$ of GDP on health (2013) and has 70 general beds per 100,000 population; hence, at least five ICU beds per 100,000 populations is a reasonably achievable goal. ${ }^{[13,14]}$ However, in MP, we are farther away from this goal, largely due to scarce resources, lack of trained human resources, and suboptimal infrastructures.

The most important human resource is nursing staff; we had dedicated nursing staff for each of the 123 ICU facilities visited. The BSc trained nurses were present in level 3 ICUs. The ratio of nurse-to-bed ratio differed according to the level of ICU care, it was on average one nurse for 3.3 beds per shift, while in level 3 ICUs, it was 1:1.7. In contrast, the Netherlands had ratio of $1: 0.8$, i.e., better than $1: 1$, UK had ratio of $1: 1$, and Sweden and France had ratio of 1:1.9. There was wide variation in staffing across Europe; $>20 \%$ of the units had ratio of $1: 1.5 .^{[15,16]}$ Such variations are due to financial budgeting as well as regional and cultural differences. Sri Lanka had nurse: patient ratio of $1: 1$ in $75 \%$ of their ICU setups whereas our best ratio was $1: 1.7$ that too in level 3 ICUs, i.e., $11.38 \% \%^{[17]}$ Infection control nurse was present only in one-third of ICU. Training courses for nurses and continuous medical education events were present in hardly $30 \%$ of the ICU setups. It is this area where our administrators should work and should ensure 1:1 nurse-to-bed ratio which in turn will improve outcomes in our ICUs. Other support staff such as physiotherapists, respiratory therapists, and ICU technician is present in very low percentage [Table 5].

Other important workforce is intensivist; the degree of intensivist varied from one facility to other. Mostly, they were from specialist of anesthesia and medicine background where they were doing operation theater and outpatient department activities. There were hardly $<10$ intensivists in the state with degree or certificate course in critical care. Almost all level 3 ICU and a few level 2, i.e., $<10$ had full-time intensivists. Out of the government setup, only two of them had dedicated critical care team with round the clock consultant cover. There are only two ISCCM certified training centers; none of the government institutes has DM training program. ${ }^{[18]}$ This is a cause of concern where the ISCCM and government should intervene to start critical care training program in the state to strengthen critical care services.

Almost all the level 3 ICU and 50\% of level 2 ICU had basics equipment for ICU setup, i.e., invasive ventilators, monitors (noninvasive and invasive), and bilevel positive airway pressure. The ventilator-to-bed ratio was 1:1 in level 3 ICUs, while on average, it was 1:3. This as compared to Sri Lanka where ventilator: bed ratio was $1: 1$ in $53 \%$ of the units. ${ }^{[17]}$ The portable ultrasound machine was available in 43/123 of the ICU, i.e., $34.9 \%$, this machine forms the lifeline in ICU apart from therapeutic interventions, this aids in early diagnosing and managing life-threatening diseases. The hemodialysis facility was present in 73/123 of the critical care facilities; apart from the level 3 ICU, none of them had a dedicated hemodialysis machine for ICU. The CRRT machine was available in eight of the total 123 facilities. Similarly, CT facility was available in $40 \%$ setups while MRI facility was hardly available. In-house ABG is must for running ICU setups, all level 3 ICU and $90 \%$ of level 2 ICU had ABG facility. There was lack of advance microbiology services in the state; this is an area of concern as good microbiology laboratory means isolation of organism which would help us target therapy. Use of broad-spectrum antibiotics without evidence would lead to isolation of drug-resistant organisms; hence, a good microbiology laboratory helps in reducing antibiotic pressure. Apart from level 3 ICU, there were no written operational procedures or disinfection policies and protocol in place; this leads to breach in standardized care of the patient. This is possible only if we train more intensivists and nurses in critical care which would help us in a protocolized approach.

In our study, quality of ICUs was heterogeneous. Most level 1 ICUs did not have a noninvasive ventilator (yet had a facility for invasive ventilation), facility for ABG, basic microbiology laboratory, and invasive monitors. Most of them are also not even obtaining central venous access. Almost all level 3 ICUs were performing tasks that are expected of such facilities. There is a clear volume-level relationship as median number of ICU beds is 6 in level 1 facilities and 24 in level 3 facilities. While we did not measure outcomes in our study, it is likely that level 3 facilities may have a better outcome compared to level 1 facilities, as a sheer function of difference in quality. ${ }^{[19]}$

Of the 123 mapped facilities, only $25 \%$ are in the public sector. Furthermore, in India, patients pay $78 \%$ of the healthcare costs as out-of-pocket expenses. ${ }^{[2]}$ This contrasts Europe where most ICU facilities are in public sector. ${ }^{[4]}$ Lack of ICUs in public facilities creates a huge economic burden when such care is required. Given more social disparities and poverty in India, proportion of ICUs in public sector needs to be higher. In our study, the most significant determinant of ICU bed in the district was its per-capita income. Since ICU care is expensive, hence, more well-off districts with a greater density of medical doctors could afford them. Economic disparity is a key determinant of accessibility to better health care, especially for critical care. 
Our study is the first study from LMIC, which describes distribution, level of care, education, and research in described ICUs. We used a scoring system to grade ICUs, which we feel is a better way to compare across regions and nations.

Our study has some lacunae; first, many attributes in an ICU were self-reported by the facility managers. We minimized this reporting bias by verifying some key attributes in an ICU. Second, we may have missed including a facility, despite using multiple overlapping sources of information. Third, we did not perform a quality assessment of ICU care based on disease outcomes or patient admission characteristics as it was beyond the scope of our study. Finally, many residents of the state may utilize ICU facilities in the neighboring states, and we were limited by geographic boundaries in our study. Despite these limitations, we believe that the current study is an important information source about ICU bed density and quality in MP.

\section{Conclusion}

Delivery of healthcare occurs at the local, regional, and national level. Yet, no planning for healthcare at either the regional or national level can occur without detailed information about the available resources. Such information about critical care facilities may be useful to identify deficiencies in the organization of care and to identify targets for level of care, infrastructure, human resource, equipment, medical education, and research. This study would eventually help in strengthening of critical services into high levels of care in the state of MP. Other states then could attempt to do mapping and in the near future will be able to get our national data of ICU Beds per 1 lac population.

\section{Acknowledgment}

We would like to acknowledge the Department of Public Health and Family Welfare, Madhya Pradesh, India.

\section{Financial support and sponsorship}

Nil.

\section{Conflicts of interest}

There are no conflicts of interest.

\section{References}

1. Amoateng-Adjepong Y. Caring for the critically ill in developing countries - Our collective challenge. Crit Care Med 2006;34:1288-9.
2. Dünser MW, Baelani I, Ganbold L. A review and analysis of intensive care medicine in the least developed countries. Crit Care Med 2006;34:1234-42.

3. WHO Centre for Health Development Ageing and Health Technical Report. A Glossary of Terms for Community Health Care and Services for Older Persons. Vol. 5. Australia: World Health Organization; 2004. p. 35.

4. Rhodes A, Ferdinande P, Flaatten H, Guidet B, Metnitz PG, Moreno RP, et al. The variability of critical care bed numbers in Europe. Intensive Care Med 2012;38:1647-53.

5. Lipman J, Lichtman AR. Critical care in Africa. North to South and the future with special reference to Southern Africa. Crit Care Clin 1997;13:255-65.

6. Bhagwanjee S. Critical care in Africa. Crit Care Clin 2006;22:433-8.

7. Baker T. Critical care in low-income countries. Trop Med Int Health 2009;14:143-8.

8. Mungekar BL. Madhya Pradesh Development Report. A Glossary of Terms for Community Health Care and Services for Older Persons. New Delhi: Academic Foundation; 2011. Available from: http://www. planningcommission.nic.in/plans/stateplan/sdr/sdr_mp1909.pdf. [Last accessed on 2016 Jan 30].

9. Medical Council of India. List of Colleges Teaching MBBS; 2015. Available from: http://www.mciindia.org/InformationDesk/ ForStudents/ListofCollegesTeachingMBBS.aspx. [Last accessed on 2015 Jan 20].

10. Rao HR. Economic Survey of Madhya Pradesh; 2011. Available from: https://www.data.gov.in/catalog/madhya-pradesh-economic-indicator. [Last accessed 2016 Jan 25].

11. Wunsch H, Angus DC, Harrison DA, Collange O, Fowler R, Hoste EA, et al. Variation in critical care services across North America and Western Europe. Crit Care Med 2008;36:2787-93, e1-9.

12. Rungta N, Govil D, Sheila N, Manish M, Divatia J, Jani CK. Guidelines Committee ISCCM. ICU Planning and Designing in India; 2010. Available from: http://www.isccm.org/images/Section1.pdf. [Last accessed on 2015 Feb 10].

13. World Bank Data. Health EXPENDITURE on GDP; 2011-2015. Available from: http://www.data.worldbank.org/indicator/SH.XPD. TOTL.ZS. [Last accessed on 2016 Jan 30].

14. World Bank Data. Hospital Beds Per 1000 People; 2011-2015. Available from: http://www.data.worldbank.org/indicator/SH.MED.BEDS.ZS. [Last accessed on 2016 Jan 14].

15. Depasse B, Pauwels D, Somers Y, Vincent JL. A profile of European ICU nursing. Intensive Care Med 1998;24:939-45.

16. Kluge GH, Brinkman S, van Berkel G, van der Hoeven J, Jacobs C, Snel YE, et al. The association between ICU level of care and mortality in the Netherlands. Intensive Care Med 2015;41:304-11.

17. Fernando J, Wickramaratne C, Dissanayake R, Kolambage Sh, Aminda M, Cooray $\mathrm{N}$, et al. Studying current status of intensive care services in Sri Lanka. Int J Crit Illn Inj Sci 2012;2:11-6.

18. IDCCM Accredited Institutions; 2017. Available from: http://www. isccm.org/idccm-overview.html. [Last accessed on 2017 May 05].

19. Kahn JM, Linde-Zwirble WT, Wunsch H, Barnato AE, Iwashyna TJ, Roberts MS, et al. Potential value of regionalized intensive care for mechanically ventilated medical patients. Am J Respir Crit Care Med 2008;177:285-91. 